\title{
Capacity Analysis of a Peripheral Nerve using Modulated Compound Action Potential Pulses
}

\author{
Michael Donohoe, Member, IEEE, Brendan Jennings, Member, IEEE, and Sasitharan Balasubramaniam, Senior \\ Member, IEEE
}

\begin{abstract}
Artificial neural stimulation of a peripheral nerve can create an in-body data communications channel. We propose the stimulation of a peripheral nerve using energy-harvesting arrays of nanodevices, embedded in biocompatible tissue patches. The resulting extracellular compound action potential (CAP) pulse can provide a data bit-stream for communicating with an embedded receiver. Our objective is to determine the maximum achievable transmission range of a CAP along a nerve and the maximum sustainable bit rate. We model the generation of a CAP and then compute the reduction in amplitude and the spreading of the pulse with propagation distance. The channel capacity is calculated for on-off keying (OOK) and digital pulse interval modulation (DPIM). We show that the transmission range depends on the number and diameters of the activated neurons contributing to the CAP amplitude and width. Our modulation analysis demonstrates the effects of attenuation, background noise, the neural refractory period and pulse broadening on the achievable bit-rate. We show how a maximum OOK bit rate of $200 \mathrm{bit} / \mathrm{s}$ can be sustained over transmission distances greater than $100 \mathrm{~mm}$. The proposed approach provides a low bit-rate, unidirectional asynchronous transmission system that could, for example, deliver simple instructions to an embedded drug-delivery system.
\end{abstract}

Index Terms-Action Potentials, Asynchronous Communication, Channel Capacity, Nanobiotechnology, Neurostimulation.

\section{INTRODUCTION}

Artificial neural stimulation uses electrical current to stimulate specific parts of the human nervous system. At present it is used to treat neurological conditions or to enhance neural connectivity for prosthetics. Stimulation may be delivered by externally powered electrodes placed on the skin surface (transcutaneous) or under the skin (subcutaneous) in closer proximity to muscles or nerves. Transcutaneous electrical nerve stimulation (TENS) has long been used for pain relief [1] while external vagus nerve stimulation is currently being investigated as a treatment for epilepsy, depression [2] and the inflammation of rheumatoid arthritis [3]. Deep brain stimulation $(D B S)$ deploys embedded electrodes to reach specific areas of the brain and is used in the treatment of Parkinson's

M. Donohoe and B. Jennings are with the Telecommunications Software \& Systems Group (TSSG), Waterford Institute of Technology, Ireland. S Balasubramaniam is with the Tampere University of Technology, Finland. Email: mdonohoe@tssg.org, bjennings@wit.ie, sasi.bala@tut.fi

This work is supported by the Academy of Finland FiDiPro programme for the project "Nanocommunications Networks" 2012 - 2016, and the Finnish Academy Research Fellow programme under Project no. 284531 It is also partly funded by the Irish Higher Education Authority under the Programme for Research in Third Level Institutions (PRTLI) cycle 5, which is co-funded by the European Regional Development Fund (ERDF), via the Telecommunications Graduate Initiative, and by Science Foundation Ireland via the CONNECT research centre (grant no. 13/RC/2077).
Disease [4]. In all cases the stimulus current is delivered by an electrode with no other electronic devices on the neural path.

A nerve can also be seen as a communications path transporting information via neurons from the body to the brain and vice versa. A whole-body communications system using touch stimuli on a finger as transmitters and brain scan information as a receiver is described by Hanisch [5]. The maximum modelled bit-rate is $40 \mathrm{bit} / \mathrm{s}$ though the detection system has a lot of background noise from other brain functions. Individual neurons transfer stimulus spikes called action potentials $(A P)$ along neuron bodies ( axons) and across neural interfaces (synapses). The published research work to date is based on single neuron analysis to model (i) molecular communications at the nanoscale between neurons or (ii) data communications along a single axon. Channel models for single-spike intraneuron and inter-neuron communications, based mainly on experimental knowledge of hippocampal neurons, have been developed by Malak and Akan [6], Balevi and Akan [7], Ramezani and Akan [8], Veletić et al [9] and Cacciapuoti et al [10]. The modeling of a multiple input, single output (MISO) neural channel is examined in more detail by Ramezani et al [11] who also consider the effects of a neural degeneratiive disease on the number of available neurotransmitters. A neuron channel model using a sub-threshold (non-spiking) stimulus was proposed by Khodaei and Pierobon [12] [13], though sub-threshold impulses have a very short range along an axon [14]. Data communications through the single median giant axon of the earthworm was modelled by Abbasi et al [15] who calculated a data throughput based on frequency shift keying (FSK). Our focus is to model a communications channel along the nervous system using multiple stimulated neurons to exploit the higher level of voltage pulse that can be generated.

An embedded transmission device could generate modulated stimulus pulses along a nerve for interpretation by an embedded receiving device. The information rate will depend on many factors such as: (i) the number and type of neurons that are stimulated; (ii) the spread in velocities of the APs; and (iii) the exact position of the detecting electrodes. The stimulus pulses should not interfere with the normal working of the nervous system and should be applied in situations where the muscle or organ at the nerve extremity was damaged and could not terminate normal nerve impulses. The receiving module could provide functionality to overcome such impairment. One potential application is the delivery of different drug types or concentrations from a programmable platform to a damaged organ, such as that described by Huang et al [16].

In previous work we modelled how ultrasound could pro- 
vide wireless energy to embedded nanowire-based devices [17] and how arrays of theses nanodevices could selectively stimulate fascicles in peripheral nerves [18]. The nanodevice array is embedded into a polymer-based patch of bio-compatible tissue [19], placed against a nerve's outer layer (Epineurium) and harvests its energy from ultrasound waves that are emitted by a portable external source. The harvested ultrasound energy is converted by the piezoelectric nanowires and releases an electronic pulse that stimulates nerve fascicles through an electrode. The pulse then is transmitted along a peripheral nerve and detected at the remote end by a receiver. The stimulated part of the nerve should be at a shallow depth under the skin to allow for maximum power harvesting. In this paper we propose the novel use of energy-harvesting nanodevice arrays as neural data transmitters and develop a novel channel model for a peripheral nerve. The receiver can be at a deeper level in the body, possibly encased by muscle and/or obscured by bone. In order to dimension such a transmission system we need to know:

- maximum number of pulses per second that a nerve can support;

- amplitude and attenuation of the pulse to determine the pulse range and intensity;

- level of noise or distortion in the nerve;

- modulation that is suited to the natural signaling properties of the nerve.

These values and parameters allow us to calculate the resulting bit rate at different distances from the stimulus point. The main contributions of our work are as follows:

- Modelling the generation and propagation of an extracellular Compound Action Potential (CAP) along a peripheral nerve subject to constraints imposed by neural biology.

- Evaluating two different modulation methods, on-off keying $(O O K)$ and digital pulse interval modulation (DPIM), that could be applied.

- Showing how a bit rate of $200 \mathrm{bit} / \mathrm{s}$ can be generated and sustained for distances over $100 \mathrm{~mm}$ despite pulse attenuation and broadening from the underlying neural channel.

The article is organised as follows: our modelling of neuron activation and action-potential generation is described in $\S \mathrm{II}$; the model of a compound action potential in §III; the channel model in $\S \mathrm{IV}$; modulation and protocols in $\S \mathrm{V}$ and our conclusions are presented in $\S \mathrm{VI}$.

\section{NEURON ACTIVATION}

The resting potential of a neuron, based on an ionic across the neural membrane, is approximately $-70 \mathrm{mV}$. If a stimulus raises this potential above $-55 \mathrm{mV}$ (e.g., by applying a pulse of magnitude $15 \mathrm{mV}$ or greater) then the neuron activates [14]. The potential across the membrane rapidly increases to about $30 \mathrm{mV}$ (a total increase of $100 \mathrm{mV}$ from rest) and an action potential $(A P)$ then propagates down the neuron's axon and transfers across a synapse to another neuron or a muscle cell. The first neuron then returns to the rest state. The following are key parameters in the neuron activation process:
TABLE I: Axon Types and Parameters

\begin{tabular}{lllll}
\hline Axon Type & Myelin & $\begin{array}{l}\text { Diameter } \\
(\mu \mathrm{m})\end{array}$ & $\begin{array}{l}\text { Velocity } \\
(\mathrm{m} / \mathrm{s})\end{array}$ & $\begin{array}{l}\text { Chronaxie } \\
(\mu \mathrm{s})\end{array}$ \\
\hline $\mathrm{A} \alpha$ & Yes & $13-20$ & $80-120$ & $50-100$ \\
$\mathrm{~A} \beta$ & Yes & $6-12$ & $35-75$ & 120 \\
$\mathrm{~A} \delta$ & Yes & $1-5$ & $10-35$ & 170 \\
$\mathrm{~B}$ & Yes & 3 & $3-15$ & 200 \\
$\mathrm{C}$ & No & $0.2-1.5$ & $0.5-2.0$ & 400 \\
\hline
\end{tabular}

- The AP cycle duration from activation to completion (typically $5 \mathrm{~ms}$ ) is called the Refractory Period $\left(T_{r e f}\right)$ and a second stimulus applied during this interval will not result in another action potential. The refractory period also ensures that an AP can only travel in one direction as the neuron will be de-activated in the opposite direction.

- The Intracellular Action Potential (IAP) is specifically the trans-membrane voltage measured from the inside of the neuron to the outside across the neural membrane and will have a maximum value of approximately $30 \mathrm{mV}$.

- The extracellular action potential or Single Fibre Action Potential (SFAP) is measured on the outside of the neuron with respect to the surrounding extracellular medium and will be much smaller in magnitude (nanovolts) than the IAP.

- The Compound Action Potential (CAP) is the algebraic sum of multiple SFAPs arising from the same external stimulus. The magnitude will depend on how many neurons are simultaneously activated.

\section{A. Activation Parameters}

The level of current needed to artificially stimulate a neuron will depend on the excitability of the neuron, the electrodeneuron distance and the stimulus duration [20]. Larger diameter neural axons are more excitable and require lower stimulus energy than smaller diameters. Such larger axons have an insulating sheath of myelin and are classed as $\mathrm{A} \alpha$, $\mathrm{A} \beta, \mathrm{A} \delta$ and $\mathrm{B}[21]$. The lowest possible stimulus current of an axon is called the rheobase. The chronaxie is the minimum time required for a stimulus current twice the value of the rheobase to stimulate a neuron [20]. Diameters, AP velocity and chronaxie value for different types of neural axons are summarised in Table I [21]. The velocity of the AP is greater in larger diameter, myelinated axons. Non-myelinated axons, classed as $\mathrm{C}$, have the smallest diameters and the lowest AP velocities. The source current intensity for stimulation must be increased as the distance between the stimulating electrode and the neurons increases. Computed values for electrode voltage and stimulus current for a range of neuron depths are shown in Table II [18] for neurons with a chronaxie of $100 \mu \mathrm{s}$. The greater the depth of penetration, the more fascicles can be reached and the more neurons can be activated.

\section{B. Intracellular and Extracellular Action Potential}

An empirical IAP model devised by Nandedkar and Stalberg [22] was based on an earlier version by Rosenfalck [23]. This model can be expressed in the space domain and the time 
TABLE II: Stimulus voltage and current for neurons with a chronaxie of $100 \mu \mathrm{s}$ at a range of depths.

\begin{tabular}{lll}
\hline $\begin{array}{l}\text { Neuron Depth } \\
(\mathrm{mm})\end{array}$ & $\begin{array}{l}\text { Electrode Voltage } \\
(\mathrm{mV})\end{array}$ & $\begin{array}{l}\text { Stimulus Current } \\
(\mathrm{mA})\end{array}$ \\
\hline 0.5 & 150.5 & 0.057 \\
1 & 204 & 0.077 \\
1.5 & 293 & 0.11 \\
2 & 419 & 0.158 \\
2.5 & 580 & 0.219 \\
3 & 777 & 0.293 \\
\hline
\end{tabular}

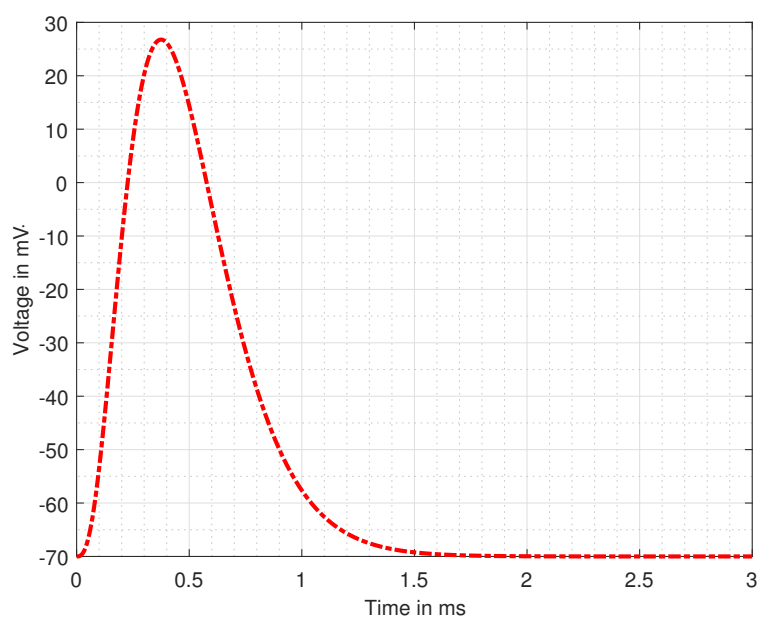

Fig. 1: Time-based intracellular action potential (IAP) model for a myelinated neuron.

domain to generate the shape and magnitude of the IAP based on experimental values. The space domain version is expressed across a distance $z$ while the time domain version uses the transform $z=v t$ where $v$ is the velocity of the IAP and $t$ is the time parameter. Three shaping parameters are used: $\alpha$ for the rising part of the IAP, $\beta$ for the exponential reduction and $\gamma$ for the resting potential. The empirical formula for the time domain IAP, $\phi_{\text {iap }}(t)$, may be written as:

$$
\phi_{i a p}(t)=\alpha t^{3} e^{-\beta t}-\gamma
$$

The time-based version produces an IAP that has a duration appropriate for a range of cellular tissue including neurons of different types. This formula was originally devised for muscle fibre with $\alpha=49152 \mathrm{mV} / \mathrm{ms}^{3}, \beta=8 / \mathrm{ms}, \gamma=90 \mathrm{mV}$ and a velocity of $4 \mathrm{~m} \cdot \mathrm{s}^{-1}$. We modify the shape of the timebased version to match the experimental shape of a myelinated neuron IAP by: i) changing the resting potential, $\gamma$, to -70 $\mathrm{mV}$, ii) setting the value of $\alpha=36864 \mathrm{mV} / \mathrm{ms}^{3}$ to generate a peak voltage of $30 \mathrm{mV}$ and iii) retaining the value of $\beta$. Our modified empirical equation is:

$$
\phi_{\text {iap }}(t)=36864 t^{3} e^{-8 t}-70
$$

A plotted example of our modelled IAP for a myelinated neuron is shown in Fig. 1. We will use this time-based IAP model in our calculation of the extracellular single fibre action potential (SFAP).

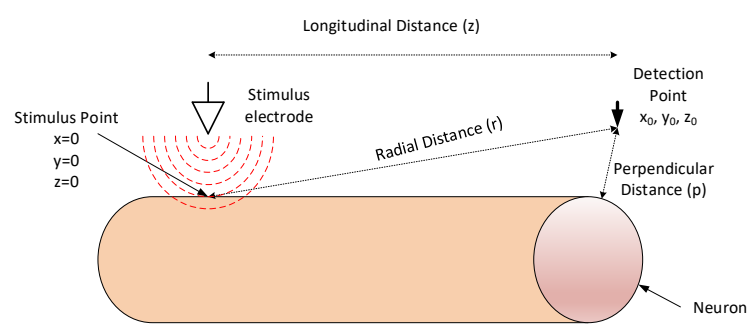

Fig. 2: Schematic view of the stimulating and detection of single fibre action potentials (SFAPs) along a single neuron.

The calculation of potential at a point in a conducting extracellular medium arising from a travelling current source is based on the principles of volume conduction [24]. The SFAP is computed from a convolution of (i) a source excitation and (ii) a weighting function based on the exact position of the detecting electrode along the fibre. If we model a single neuron as a long thin cylinder then we can define the direction of propagation of an SFAP as along the $z$ axis and the initial excitation point as $(0,0,0)$. The detection point for the SFAP is outside the fibre at location $\left(x_{0}, y_{0}, z_{0}\right)$ in the extracellular domain as shown in Fig. 2. The extracellular potential at this point will vary with the radial distance from the propagating source of excitation [14]. The radial distance will vary specifically with the longitudinal propagation distance and can be expressed as $r(z)$ once the detection point is a fixed perpendicular distance $p$ (in the $x y$ plane) from the fibre. The potential at a specific time can be expressed as an integration of the contributions of all transmembrane current sources of length $d z$. Two types of model have been developed for SFAPs: monopole models based on transmembrane current (second derivative of the IAP) and dipole models based on dipole moment (first derivative of the IAP). Monopole models were developed by Plonsey [25] and Nandedkar and Stalberg [22] and a dipole model was developed by Dimitrova et al [26]. These were analysed by Falces et al [27] who noted that the dipole model gave better results at boundaries and was more consistent with experimental data. We will use a dipole model in our derivation of the SFAP and our formulation is based on the equation derived by Falces [27]. The extracellular potential at time $t$, $\phi_{e}(t)$, for a neuron of radius $a$ can be modelled as the output signal of a linear system and computed as the convolution of two time dependent functions:

$$
\phi_{e}(t)=\frac{a^{2} \sigma_{i}}{4 \sigma_{e} v} \frac{\partial \phi_{i a p}(t)}{\partial t} * \frac{\partial \frac{1}{r_{v}(t)}}{\partial t}
$$

The intracellular conductivity is $\sigma_{i}$, , the extracellular conductivity is $\sigma_{e}$ and the conduction velocity is $v$. The radial distance, $r_{v}(t)$, can be expressed as:

$$
r_{v}(t)=\sqrt{\left(z_{0}-v t\right)^{2}+p^{2}}
$$

This distance will be at minimum when the IAP reaches the longitudinal distance $\left(z_{0}\right)$ of the electrode from the source. The SFAP conduction velocity, $v$, is also proportional to the diameter of a neuron, $d(=2 a)$, and can be expressed as:

$$
v=h d
$$




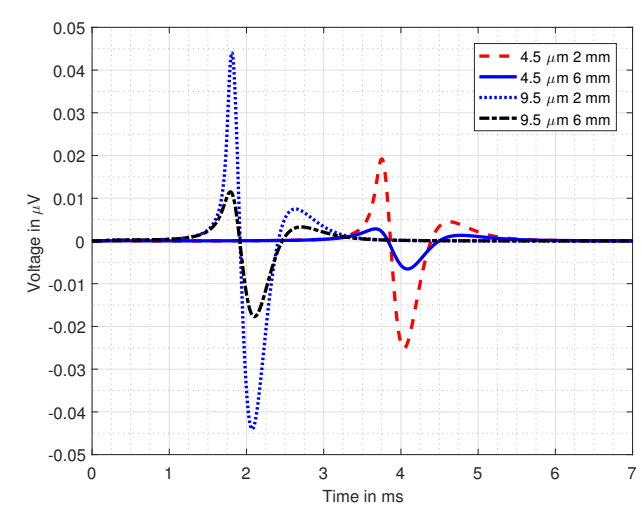

(a) SFAPs for a $4.5 \mu \mathrm{m}$ diameter neuron and $9.5 \mu \mathrm{m}$ diameter neuron at electrode perpendicular distances of $2 \mathrm{~mm}$ and $6 \mathrm{~mm}$.

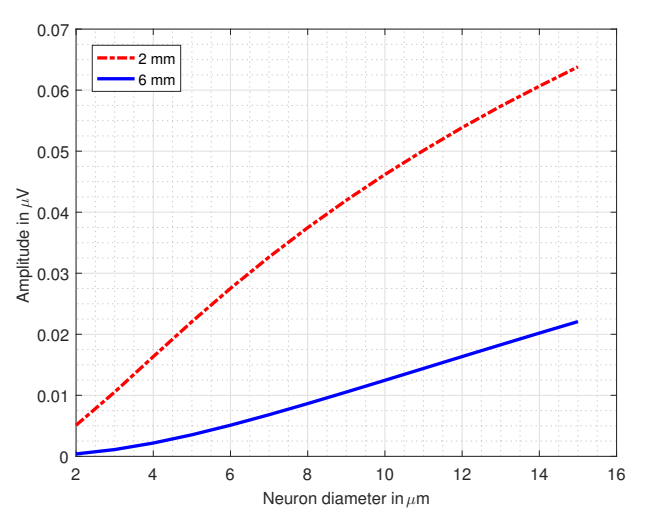

(b) SFAP amplitude (positive peak) plotted against a range of neuron diameters for two different detection electrode perpendicular distances.

Fig. 3: Variation of SFAP amplitude with neuron diameter and detection electrode perpendicular distance at a fixed longitudinal distance of $100 \mathrm{~mm}$.

The constant of proportionality, $h$, is approximately 6 for myelinated neurons when the velocity is expressed in $\mathrm{m} / \mathrm{s}$ (or $\mathrm{mm} / \mathrm{ms}$ ) and the diameter is expressed in $\mu \mathrm{m}$ [28]. We fix the value of extracellular conductivity, $\sigma_{e}$, at $0.3 \mathrm{~S} / \mathrm{m}$ and the intracellular conductivity, $\sigma_{i}$, at $1 \mathrm{~S} / \mathrm{m}$ [29]. Our modelled example of SFAPs for myelinated neurons of two different diameters is shown in Fig. 3a. The duration of the SFAP is the same in each case (approximately $2.55 \mathrm{~ms}$ ) but the time taken to reach the detection point is longer for the lower diameter (and lower velocity) neuron. The recorded SFAPs are tri-phasic with both positive and negative peaks. The amplitudes of the SFAP peaks depend not only on the relative positions of the source and detecting electrode but also on the conductivity ratio between the intracellular medium and the extracellular medium, as shown in (3). The relationship between SFAP amplitude (for positive/negative peaks) and neuron diameter is approximately linear for the range of diameters that we are studying [28] and is shown for the larger positive peak in Fig. 3b. The highest frequency for generating SFAPs is limited by the refractory period (up to $5 \mathrm{~ms}$ ) and will be approximately 200 pulses/sec.

\section{Compound Action Potential}

An SFAP for a single neuron is difficult to detect because of its low amplitude and the possibility of interference from other neurons. However, a stimulus pulse of sufficient strength will trigger multiple SFAPs in one or more fascicles. The SFAPs will sum algebraically to give a compound action potential $(C A P)$ which is easier to detect by an extracellular receiver because of the higher summed voltage although the peak voltage may still be of the order of microvolts or low millivolts.

\section{A. CAP Simulation}

We model CAP measurement over multiple neurons in a similar way to that shown for a single neuron in Fig. 2. The peripheral nerves that we are considering (sural, median, radial) are elliptical or circular in cross-section with average radii ranging from $1.7 \mathrm{~mm}$ (median) to $1.5 \mathrm{~mm}$ (sural). The minimum perpendicular detection distance occurs when the detecting electrode is on, or very close to, the surface of the nerve. The neurons are at varying distances from the detection point with corresponding differences in SFAP amplitudes. Wijesinghe et al, [30] show the average distance of neurons from the centre of the nerve can bet set at a value of 0.66 of the nerve radius without loss of accuracy in CAP calculation. We adapt this model with the neurons concentrated at the centre of the nerve and the detecting electrode set at slightly greater than the typical peripheral nerve radius. A minimum perpendicular detection distance of $2 \mathrm{~mm}$ ensures that the nerve dimensions and neuron distances are properly accounted for in simulating multiple SFAPs without overestimating the magnitude of the resulting CAP. The duration (width in time) of a CAP will depend on what fraction of the neurons in the fascicles are stimulated and the velocity of propagation of the SFAPs. Schoonhoven et al [31] used a volume conduction and convolution model, similar to the one we described in §II-B, to compute SFAPs and then combined these mathematically into a CAP. Models for CAPs were also developed by Wijesinghe et al, [30] based on similar principles. The general formulation states that a CAP at a time $t$ and distance $l$ from the stimulating electrode may be expressed as:

$$
C A P(t, l)=\sum_{j=1}^{N} \phi_{e j}\left(t-\tau_{j} ; v_{j}\right)
$$

There are $N$ active fibres in total in the nerve and $\phi_{e j}$ is the SFAP of the $j$ th neuron. The conduction velocity is $v_{j}$ and is usually constant for a given neuron. The arrival time of the stimulus at the detection point, $\tau_{j}$, is derived from the distance and the SFAP velocity :

$$
\tau_{j}=\frac{l}{v_{j}}
$$

If the neurons are of different diameter then the SFAPs will have different velocities and the CAP at distance $l$ will show spreading from the variation in SFAP arrival times. The CAP amplitude will reduce with longitudinal distance because the underlying multi-speed SFAP bi-phasic peaks will progressively start to cancel each other out [30] giving a 


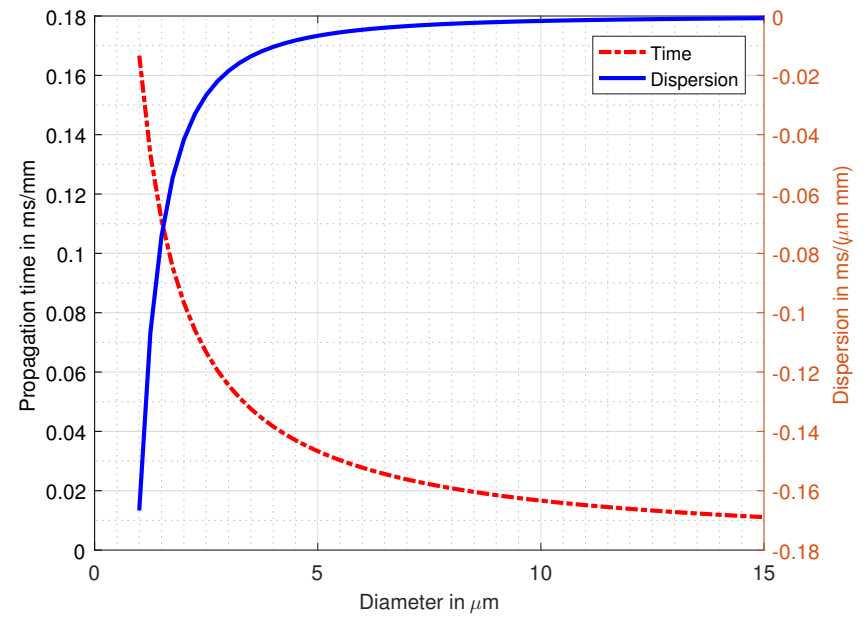

Fig. 4: Spread of arrival times of SFAPs in $\mathrm{ms} / \mathrm{mm}$ for varying neuron diameters. The dispersion in $\mathrm{ms} / \mu \mathrm{m} / \mathrm{mm}$ is also shown.

reduced summation. A plot of propagation times for SFAPs over a range of diameters (and hence velocities) is shown in Fig. 4 for a distance of one mm. We compute the dispersion, $D$, as the rate of change of propagation time $t$ with respect to neuron diameter $d$ and distance $z$ at any given point as follows:

$$
D=\frac{1}{z} \frac{\mathrm{d} t}{\mathrm{~d} d}
$$

Dispersion is expressed per unit length as $\mathrm{ms} / \mu \mathrm{m} / \mathrm{mm}$. Each neuron diameter has its own value of dispersion but it is possible to use a single value of dispersion over a narrow range of diameters to simplify calculation of pulse spread. This spreading in time of a CAP is analogous to the dispersion that occurs in an optical fibre when a propagating optical pulse contains a range of wavelengths [32] that travel at slightly different velocities. A nerve or fascicle with a greater proportion of smaller diameter neurons and lower conduction velocities will generate a CAP with a lower amplitude and with greater spreading over distance than a fascicle with a greater proportion of larger diameter neurons. We can illustrate this by modelling a nerve with a total of 10,000 neurons, similar to the sural nerve model used by Stegeman et al [33]. A peripheral nerve of this type has multiple fascicles with a bimodal spread of neural diameters around $4.5 \mu \mathrm{m}$ and $9.5 \mu \mathrm{m}$.

We use this distribution to generate three different CAPs: (i) a bimodal CAP from all the neuron diameters, (ii) a single mode CAP from the distribution of lower speed neurons with smaller diameters and (iii) a single mode CAP from the higher speed neurons with larger diameters. The resulting CAPs are generated by summing all the calculated SFAPs and are shown in Fig. 5. We fix the perpendicular distance of the detection electrode at $2 \mathrm{~mm}$. A single mode CAP can be generated from higher velocity SFAPs if the stimulus pulse intensity and duration are set to stimulate larger diameter neurons in a fascicle. A more intense and longer duration stimulus pulse will stimulate both larger and smaller diameter neurons creating a bimodal CAP with higher velocity and lower velocity SFAPs. The bimodal CAP shows that the larger

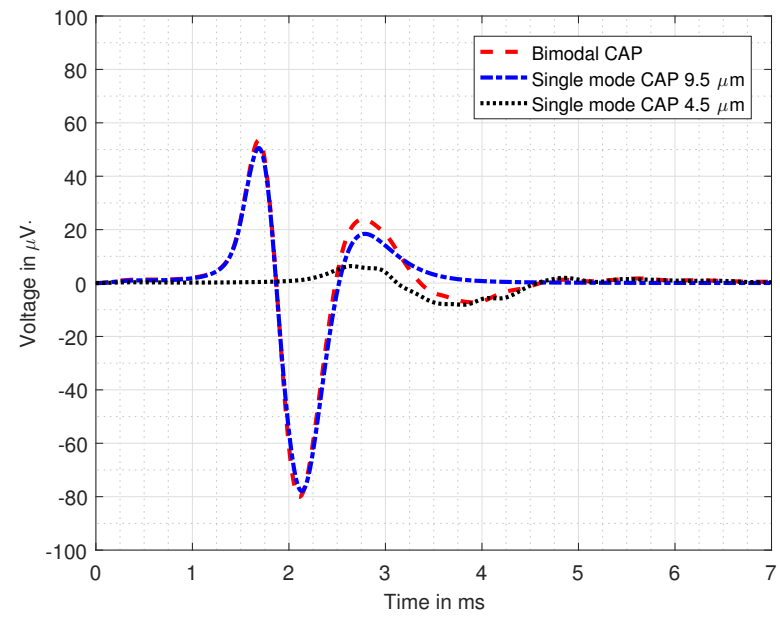

Fig. 5: Bimodal CAP of nerve and single mode CAPs of fascicles with mean diameters $4.5 \mu \mathrm{m}$ (6000 neurons) and 9.5 $\mu \mathrm{m}$ (4000 neurons) measured at a longitudinal distance of 100 $\mathrm{mm}$.

diameter neurons dominate even though numerically there are more neurons of lower diameter. The change in amplitude (positive and negative peaks) and duration (spread) of a CAP will be important parameters in assessing the use of CAPs as pulses for data transmission.

\section{B. CAP Data Pulses}

A data transmission system requires a data source, transmission channel and a receiver. In our model an external source will convert data into coded ultrasound stimulus pulses for transmission to an embedded nanodevice patch [18]. The patch will stimulate fascicles in a nerve and create a corresponding stream of coded CAPs. A receiver at the distant end must detect these CAPs, record them and interpret them using some decoding algorithm. We will use the positive peak of a single mode CAP as a measure of the amplitude of a pulse. This peak will decline with distance and the CAP will broaden. We now define an equivalent data pulse format primarily to simplify our computations while retaining all the attributes of a CAP.

- We model the CAPs using Gaussian Pulses to replicate the attributes of CAPs (decreasing amplitude, increasing width) as they propagate along a nerve without contravening the limit set by the refractory period.

- The Amplitude is based on a mean neuron diameter $\bar{d}$ and a standard deviation in diameter $\sigma_{d}$.

- The Width of the Gaussian pulse in time is a standard deviation based on the spread of propagation velocities of the constituent SFAPs.

- The Symbol Rate of the nerve can be calculated within the constraints of the refractory period and the intersymbol interference (ISI) caused by pulse spreading.

- The maximum possible Shannon Capacity of the nerve is computed from the symbol rate and the signal-tonoise ratio $(S N R)$ of the nerve. The actual capacity will 
depend on constraints like the refractory period and the modulation method.

We evaluate our neural transmission system by modelling two single mode CAPs as Gaussian pulses. The amplitude is based on the average of a number of summations of SFAP positive peaks for (i) 6000 neurons with a mean diameter, of $4.5 \mu \mathrm{m}$ and a standard deviation in diameter of $1 \mu \mathrm{m}$ and (ii) 4000 neurons with a mean diameter of $9.5 \mu \mathrm{m}$ and a standard deviation in diameter of $1 \mu \mathrm{m}$. The equation for our Gaussian pulse voltage with respect to time, $V(t)$, is as follows:

$$
V(t)=A_{\max } e^{\frac{-\left(t-\mu_{c a p}\right)^{2}}{2 \sigma_{c a p}}}
$$

The peak amplitude of the Gaussian pulse CAP (from the summed SFAPs) at the point of measurement is the positive peak $A_{\max }$. The time (in ms) at which the peak amplitude is detected at the fixed detection point provides the mean, $\mu_{\text {cap }}$, of the modelled pulse. The width of a CAP in time has a minimum value set by the width of the underlying SFAPs $(2.55 \mathrm{~ms})$. Our equivalent Gaussian pulse replicates this minimum value with a core standard deviation of one-sixth of this width $(0.425 \mathrm{~ms})$. The total standard deviation in time of the modelled CAP, $\sigma_{c a p}$, is calculated from two components: (i) a core standard deviation of $\sigma_{0}=0.425 \mathrm{~ms}$ added to (ii) the broadening due to dispersion calculated from the standard deviation of the underlying diameters (and hence velocities) at any time $t$. We can calculate the pulse broadening, $\Delta P$, over a length $z$ due to dispersion as follows:

$$
\Delta P=D_{\bar{d}} \sigma_{d} z
$$

The dispersion of the mean diameter is $D_{\bar{d}}$ (see $\S$ III) and the standard deviation of the diameter range is $\sigma_{d}$. The modelled CAP Gaussian pulse will then have a total standard deviation (in $\mathrm{ms}$ ) as follows:

$$
\begin{aligned}
\sigma_{c a p} & =\Delta P+\sigma_{0} \\
& =D_{\bar{d}} \sigma_{d} z+\sigma_{0} \\
& =D_{\bar{d}} \sigma_{d} z+0.425
\end{aligned}
$$

This ensures that the width of the modelled Gaussian pulse, a combination of the underlying CAP width plus the broadening due to dispersion, is comparable to the width of the corresponding simulated CAP. Examples of CAPs modelled as a Gaussian pulses are shown in Fig 6. The peak amplitude, $A(z)$, over a range of propagation distances for a fascicle with $k$ neurons can be averaged over a number of simulations and modelled by an empirical exponential function as follows:

$$
A_{k}(z)=G_{k} e^{-\alpha_{k} z}
$$

The empirical parameters $G_{k}$ and $\alpha_{k}$ will have values that depend on the mean neuron diameter, the standard deviation of the diameters and the number of neurons in the fascicle. The reduction in amplitude and the increase in spread of our modelled CAPs as the detection distance increases is shown in Fig. 7. We now examine how a stimulated stream of CAPs can be used for data communications.

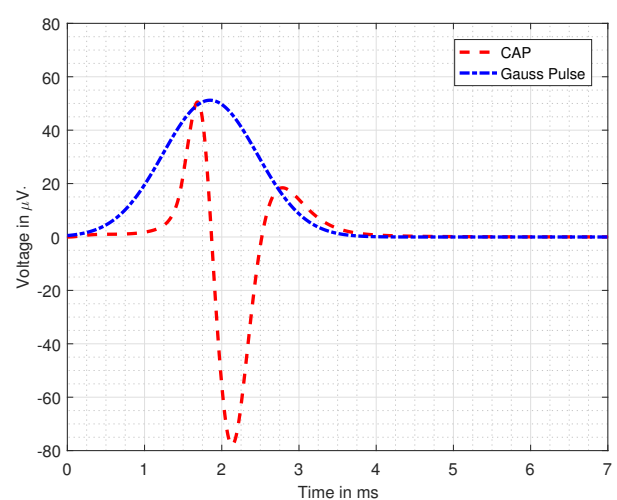

(a) Gaussian pulse compared with equivalent CAP at a detection distance of $100 \mathrm{~mm}$.

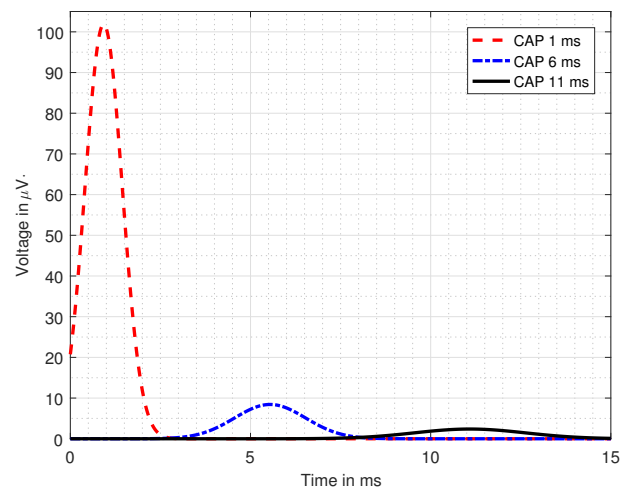

(b) Gaussian CAP pulse showing amplitude reduction and pulse spreading at time intervals of $1 \mathrm{~ms}, 6 \mathrm{~ms}$ and $11 \mathrm{~ms}$.

Fig. 6: Gaussian pulses modelled on a CAP generated from 4000 neurons with a mean diameter of $9.5 \mu \mathrm{m}$ and standard deviation of $1 \mu \mathrm{m}$

\section{Neural Data Channel}

We model CAPs as symbols to convey information in bits. The capacity of the system is determined by (i) the symbol rate (symbols per second), (ii) the number of different symbols and (iii) the number of bits that each symbol represents. The maximum possible symbol rate is limited to $200 / \mathrm{s}$ by the total refractory period, $T_{r e f}$, if a normal level of stimulus is used (see §II). Pulse spreading increases the probability of intersymbol interference (ISI) and is countered by reducing the symbol rate. The basic symbol rate for any Gaussian pulse is calculated using a peak-to-peak time difference at the point of detection of approximately four times the standard deviation $\left(4 \sigma_{c a p}\right)$. If we compare $T_{r e f}$ with $4 \sigma_{c a p}$ we get the following expressions for symbol rate $S_{c a p}$ :

$$
\begin{aligned}
& S_{\text {cap }}=\frac{1}{T_{r e f}}, T_{\text {ref }} \geq \frac{1}{4 \sigma_{c a p}} \\
& S_{\text {cap }} \approx \frac{1}{4 \sigma_{c a p}}, T_{\text {ref }}<\frac{1}{4 \sigma_{c a p}}
\end{aligned}
$$

The symbol rate will be in symbols/s if $\sigma_{c a p}$ is expressed in seconds. By substituting (11) we can calculate the effect of 


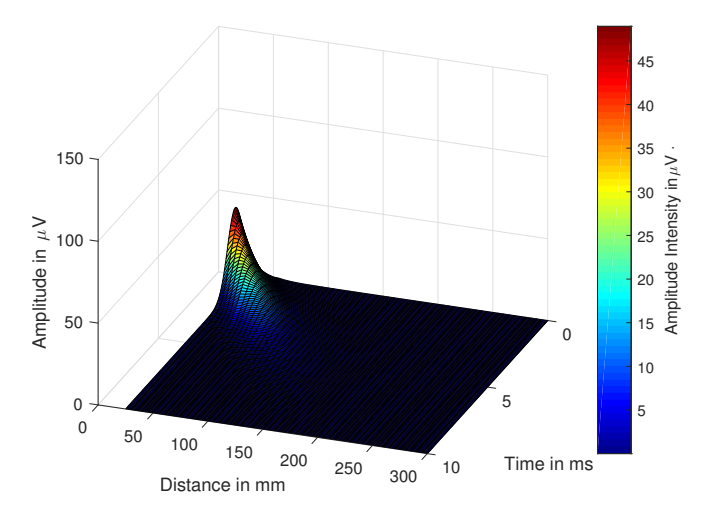

(a) CAP for 6000 neurons with a mean diameter of $4.5 \mu \mathrm{m}$.

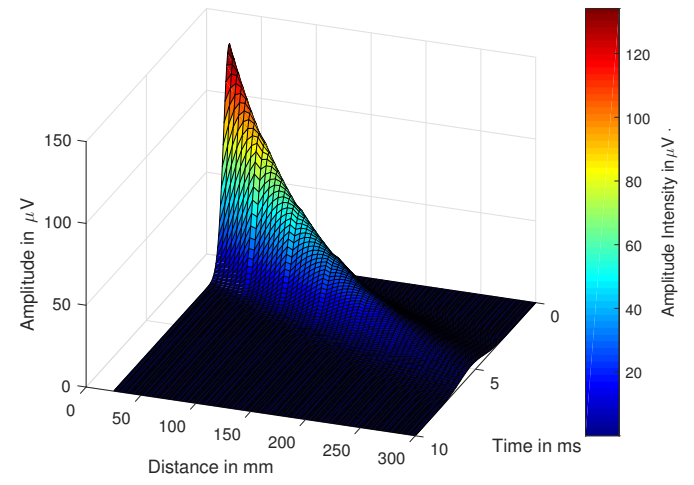

(b) CAP for 4000 neurons with a mean diameter of $9.5 \mu \mathrm{m}$.

Fig. 7: Gaussian pulse model of CAPs showing decrease in amplitude and increase in pulse spread with distance and time.

pulse spreading on the symbol rate:

$$
\begin{aligned}
S_{c a p} & \approx \frac{1}{4\left(D_{\bar{d}} \sigma_{d} z+\sigma_{0}\right)}, T_{r e f}<\frac{1}{4 \sigma_{c a p}} \\
& \approx \frac{1}{4\left(D_{\bar{d}} \sigma_{d} z+0.425\right)}
\end{aligned}
$$

The symbol rate crossover points from $T_{r e f}$ to $4 \sigma_{c a p}$ for our modelled CAPs are shown in Fig. 8. The symbol rate itself does not define the channel capacity as the presence of noise in a channel will dictate the number of symbols, $M$, and the number of bits per symbol $\left(\log _{2} M\right)$ that the channel can support.

\section{A. Channel Capacity}

We assume our multi-neuron path has average white Gaussian noise $(A W G N)$ with root mean square $(r m s)$ values in the range $5 \mu V$ to $10 \mu V$ [34] [35]. This is in accordance with models for neural noise based on experimental microneurographic measurements of normal (as opposed to stimulated) CAPs [36] [37]. Some models for single neuron AP generation also assume Gaussian noise [7] though others propose the addition of some non-Gaussian frequency-dependent noise [38] to provide more accuracy in single-spike capacity and

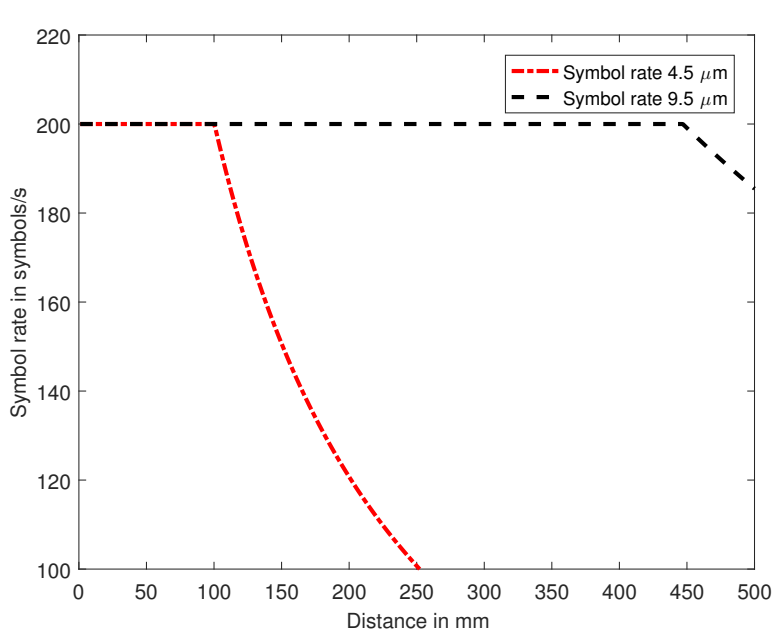

Fig. 8: Symbol rates for a CAP of 6000 neurons with a mean diameter of $4.5 \mu \mathrm{m}$ and a CAP of 4000 neurons with a mean diameter of $9.6 \mu \mathrm{m}$. The symbol rate drops at the point where the inter-pulse interval exceeds the refractory period.

sorting models. Amplitude reduction of the CAP with distance, $z$, will lower the signal-to-noise ratio $(S N R)$ and hence reduce maximum channel capacity. The SNR is derived from the noise level rms $\sigma_{n}$ and the amplitude of the CAP $A(z)$ (12) as follows:

$$
\begin{aligned}
\operatorname{SNR}(z) & =\frac{A(z)^{2}}{\sigma_{n}{ }^{2}} \\
& =\frac{\left(G_{k} e^{-\alpha_{k} z}\right)^{2}}{\sigma_{n}{ }^{2}}
\end{aligned}
$$

The SNR is a simple ratio but it can also be expressed in decibels $(d B)$ as follows:

$$
S N R_{d B}(z)=10 \log _{10}\left(\frac{\left(G_{k} e^{-\alpha_{k} z}\right)^{2}}{\sigma_{n}{ }^{2}}\right)
$$

The SNR decreases with both distance, as a result of amplitude reduction, and with increasing noise level. The maximum possible capacity, $C$ bit/s, of a noisy channel with bandwidth $U$ is given by Shannon's formula [39]:

$$
C=U \log _{2}(1+S N R)
$$

In this case the bandwidth will be the symbol rate, $S_{c a p}$, divided by 2 [40]. Substituting (13), (14) and (15) we get

$$
C_{c a p}(z)=\left\{\begin{array}{r}
\frac{1}{2 T_{r e f}} \log _{2}\left(1+\frac{\left(G_{k} e^{-\alpha_{k} z}\right)^{2}}{\sigma_{n}{ }^{2}}\right), \\
\text { if } T_{r e f} \geq \frac{1}{4 \sigma_{c a p}} \\
\frac{1}{4\left(D_{\bar{d}} \sigma_{d} z+0.425\right)} \log _{2}\left(1+\frac{\left(G_{k} e^{-\alpha_{k} z}\right)^{2}}{\sigma_{n}{ }^{2}}\right), \\
\text { if } T_{r e f}<\frac{1}{4 \sigma_{c a p}}
\end{array}\right.
$$




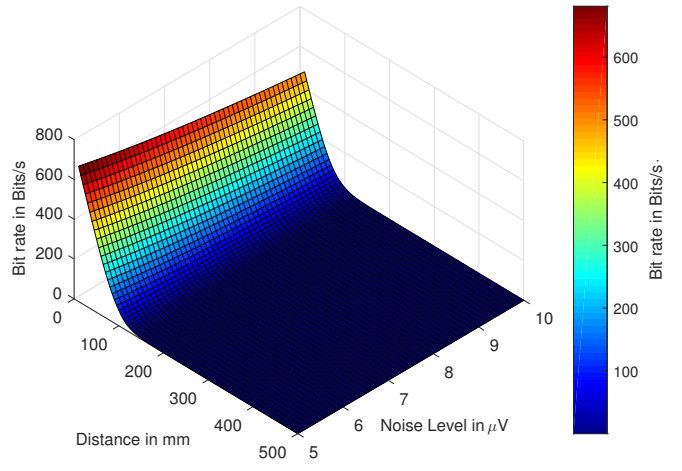

(a) Channel capacity CAP $(4.5 \mu \mathrm{m})$.

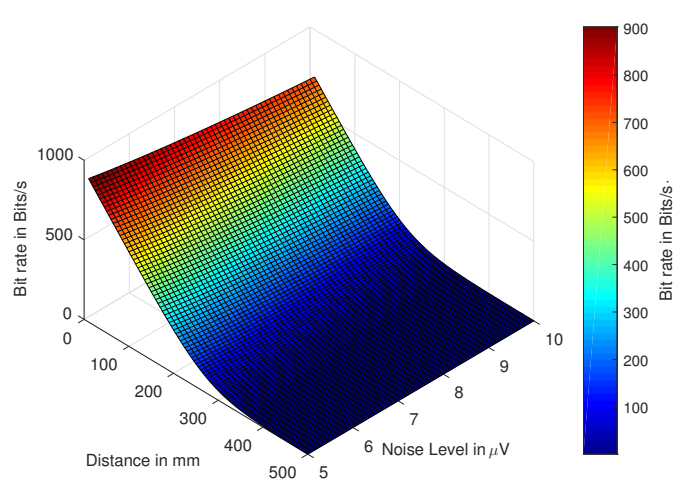

(b) Channel capacity CAP $(9.5 \mu \mathrm{m})$.

Fig. 9: Capacity plots for (a) CAP of 6000 neurons with a mean diameter of $4.5 \mu \mathrm{m}$ and (b) a CAP of 4000 neurons with a mean diameter of $9.5 \mu \mathrm{m}$.

The equations shows that the channel capacity for a nerve or fascicle at any given distance $z$ from the stimulus point depends on:

- the refractory period of the neurons, $T_{\text {ref }}$;

- the number of neurons $k$ and the mean diameter (determining the CAP amplitude);

- the background neural noise level $\left(\sigma_{n} ;\right)$

- the spread of neuron diameters around the mean value $\left(\sigma_{d}\right)$;

- the dispersion of the mean neuron diameter $\left(D_{\bar{d}}\right)$.

This theoretical capacity will be greater than or equal to the refractory pulse limit of 200/s as long as the SNR is greater than or equal to $3(4.77 \mathrm{~dB})$. The Shannon calculation gives the maximum possible bit rate with noise, as shown in Fig. 9, but does not define the type of modulation (number of different symbols and bits per symbol) that would be needed to achieve these bit rates. The number of symbols, $M$, will depend on the SNR as follows:

$$
\begin{aligned}
M & =\sqrt{(1+S N R)} \\
& =\sqrt{\left(1+\frac{\left(G_{k} e^{-\alpha_{k} z}\right)^{2}}{\sigma_{n}{ }^{2}}\right)}
\end{aligned}
$$

The higher the SNR, the greater the channel capacity but more symbols must be created to achieve that capacity. The number of bits per symbol, $b_{s}$, is:

$$
\begin{aligned}
b_{s} & =\log _{2}(M) \\
& =\frac{1}{2}\left(\log _{2}\left(1+\frac{\left(G_{k} e^{-\alpha_{k} z}\right)^{2}}{\sigma_{n}^{2}}\right)\right)
\end{aligned}
$$

If there is a limit on the number of symbols that can be employed, there will be a consequent limit on the number of bits per symbol and the result will be a reduced capacity. We now examine what data modulation methods can be applied to these intermittent baseband CAP pulses to compute the attainable bit rate.

\section{Modulation Methods and Communications PROTOCOLS}

The amplitude of a CAP will depend on the number of neurons that are activated and is subject to statistical variation. It would be difficult to encode information using stepped amplitude levels so pulse amplitude modulation (PAM) is not considered. The width of a CAP is also subject to some statistical variation and cannot be varied systematically at source so we do not consider pulse width modulation (PWM). Although a CAP has both positive and negative peaks, these are produced simultaneously and it is not possible to use the positive peak to represent a logic " 1 " and a negative peak to represent a logic " 0 ". Consequently the encoding will be unipolar using the presence or absence of a pulse to encode information. Two different encoding methods are now discussed.

- Digital Pulse Interval Modulation (DPIM) uses timed intervals between pulses as symbols in order to transmit a data value. The inter-pulse interval is sub-divided into timeslots and the value (in bits) is determined by the number of timeslots. The detection point must correctly calculate the number of timeslots between pulses in order to avoid data errors.

- On-off Keying $(O O K)$ would send a voltage pulse to represent a binary " 1 " and no pulse (for the same time duration as one pulse) to represent a binary " 0 ". This represents two symbols $(M=2)$ with one bit per symbol. There are two variants: (i) unipolar non-return to zero (NRZ) where 1 and 0 are of similar duration and unipolar (ii) return to zero (RTZ) where each positive pulse (1) returns to a zero level for part of the timed duration.

\section{A. Modulated Bit Rate}

DPIM has been proposed as a coding system for optical wireless (non-fibre based) communications by Ghassemlooy et al [41] who compare DPIM throughput, efficiency and error performance with both OOK and pulse position modulation $(P P M)$. Versions of DPIM for super-slow bacterial molecular communications have been proposed by Krishnaswamy et al [42] (Time Elapse Communication or TEC) and Barros et al [43] (Dynamic Time-Slot Configuration with Silent Communication). MacKay and McCulloch [44] explored the throughput that could be achieved in a single neuron using OOK and DPIM and we base our CAP DPIM calculations on their work. 
TABLE III: DPIM parameters.

\begin{tabular}{lllllll}
\hline $\begin{array}{l}T_{\text {ref }} \\
(\mathrm{ms})\end{array}$ & $\begin{array}{l}\Delta t \\
(\mathrm{~ms})\end{array}$ & $\begin{array}{l}T_{\max } \\
(\mathrm{ms})\end{array}$ & Symbols & Bits/symbol & Bits/s & Achievable \\
\hline 5 & 5 & 30 & 4 & 2 & 133 & Yes \\
5 & 2.5 & 25 & 6 & 2.58 & 206 & No \\
5 & 1.66 & 21.66 & 7 & 2.8 & 258 & No \\
5 & 1.25 & 20 & 8 & 3 & 300 & No \\
\hline
\end{tabular}

In our variant of DPIM coding, one information symbol is represented by a CAP pulse plus the time interval until the next CAP $\left(T_{s}\right)$. A maximum and minimum duration is set on this inter-pulse interval $\left(T_{\max }, T_{\min }\right)$ and it is then sub-divided into timeslots of a set length $(\Delta t)$. The number of symbols, $M$ will be:

$$
M=\frac{T_{\max }-T_{\min }}{\Delta t} .
$$

The symbol size $n$ will vary with $T_{s}$ and will range between 1 and $M$ timeslots. The mean value of $T_{s}$, if all $M$ values are used equally frequently, will be $\frac{1}{2}\left(T_{\max }+T_{\min }\right)$ and the average symbol rate, $S_{a v g}$, will be:

$$
S_{a v g}=\frac{2}{T_{\max }+T_{\min }} .
$$

The number of bits per symbol will be $\log _{2}(M)$. The minimum value, $T_{m i n}$, is the total refractory period, $T_{\text {ref }}$. We choose the timeslot length $\Delta t$ as the minimum time to distinguish between two consecutive modelled Gaussian pulses $\left(4 \sigma_{c a p}\right)$. Substituting from (11) and (21) we calculate the average bit rate, $B_{a v g}$, in the absence of noise using the average symbol rate and the number of bits per symbol:

$$
\begin{aligned}
B_{a v g} & =\frac{2}{T_{\max }+T_{\min }} \log _{2}\left(\frac{T_{\max }-T_{\min }}{\Delta t}\right) \\
& =\frac{2}{T_{\max }+T_{\text {ref }}} \log _{2}\left(\frac{T_{\max }-T_{r e f}}{4 \sigma_{\text {cap }}}\right) \\
& =\frac{2}{T_{\max }+T_{\text {ref }}} \log _{2}\left(\frac{T_{\max }-T_{r e f}}{4\left(D_{\bar{d}} \sigma_{d} z+0.425\right)}\right)
\end{aligned}
$$

The optimum value of $T_{\max }$ is found by iteration once values are assigned to $T_{r e f}$ and $\Delta t$. The values are shown in Table III. The parameter $\Delta t(\mathrm{~ms})$ increases in value with distance and the number of bits per symbol drops. If we assume binary coding then the number of symbols must be a power of 2 and the number of bits per symbol must be an integer. The only achievable value within the limits set by our model is 4 symbols at 2 bits per symbol as shown in Table III. This results in an average bit rate of $133 \mathrm{bit} / \mathrm{s}$, inferior even to the $200 \mathrm{bit} / \mathrm{s}$ refractory limit ceiling. Consequently, we do not consider DPIM suitable for neural data transmission in our model. Instead we will examine in more detail the performance of OOK.

The OOK bit rate is the same as the symbol rate and is a maximum of $200 \mathrm{bits} / \mathrm{s}$. This rate can be sustained until either (i) the SNR drops below 3 or (ii) pulse broadening changes the symbol rate. The crossover point for pulse broadening has been illustrated in Fig 8. The reduction in OOK bit rate caused by a falling SNR is plotted in Fig. 10. Our results show that CAP amplitude reduction and SNR have a much greater effect on

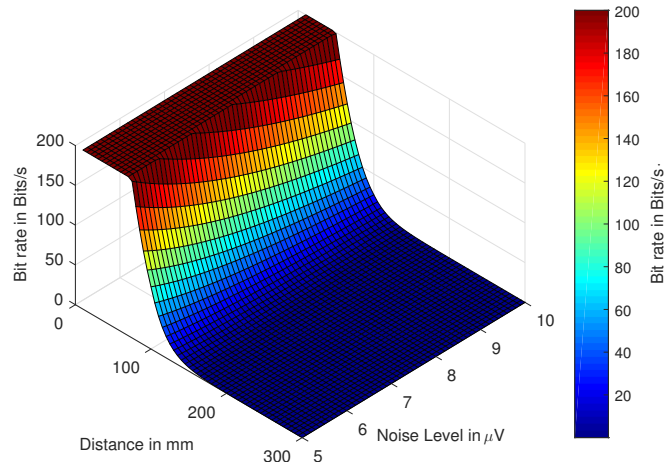

(a) OOK bit rate for CAP of $4.5 \mu \mathrm{m}$.

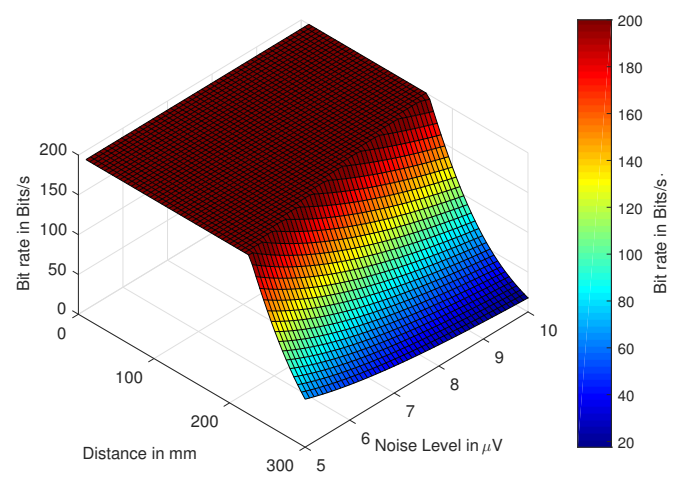

(b) OOK bit rate for CAP of $9.5 \mu \mathrm{m}$.

Fig. 10: OOK bit rates for (a) CAP of 6000 neurons with a mean diameter of $4.5 \mu \mathrm{m}$ and (b) a CAP of 4000 neurons with a mean diameter of $9.5 \mu \mathrm{m}$.

reducing the OOK $200 \mathrm{bit} / \mathrm{s}$ transmission distance than pulse broadening (Fig. 10).

The OOK bit error rate (BER) measures the probability of data detection errors occurring according as the SNR decreases. The equation for OOK BER is based on the complementary error function or erfc [45], the probability that a " 0 " is detected instead of a " 1 " or vice versa. The total probability of bit error is calculated by applying the erfc to the derivation of SNR from (15) as follows:

$$
\begin{aligned}
B E R & =\frac{1}{2}(\operatorname{erfc}) \sqrt{\frac{A(z)^{2}}{\sigma_{n}^{2}}} \\
& =\frac{1}{2}(\operatorname{erfc}) \sqrt{\frac{\left(G_{k} e^{-\alpha_{k} z}\right)^{2}}{\sigma_{n}^{2}}}
\end{aligned}
$$

The OOK bit error rate (BER) for noise levels of $5 \mu \mathrm{V}$ and 10 $\mu \mathrm{V}$ is shown in Fig. 11. The plot shows how BER increases as the SNR decreases.

\section{B. Channel Data-level Communications Protocol}

The modelled neural CAP communications channel is serial, unidirectional, low bit rate and therefore suitable for asynchronous transmission, where the sender and receiver have separate clocks. Asynchronous data link messages usually 


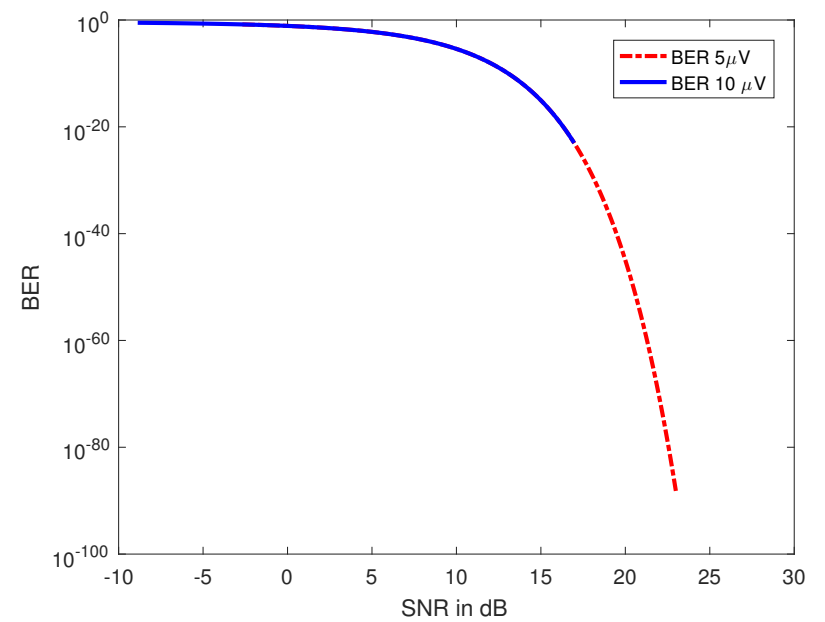

Fig. 11: BER for OOK for modelled 9.5 $\mu \mathrm{m}$ CAP with noise levels of $5 \mu \mathrm{V}$ and $10 \mu \mathrm{V}$.

have a start bit, a character coded as pulses (typically 8 bits), an optional parity bit and finally a stop bit. The send and receive clocks are both based on the expected bit rate and can differ by up to $5 \%$ without mis-interpreting the last bit of a ten-bit sequence. One low bit rate protocol in current use is the unidirectional, asynchronous X10 protocol for addressing and sending commands to simple electrical appliances (e.g. lights) over in-house power lines [46]. The highest possible transmission rate is $50 \mathrm{bits} / \mathrm{s}$. The protocol transmits a four bit start-code ( $\left.\begin{array}{llll}1 & 1 & 1 & 0\end{array}\right)$, a four bit "house code" device address followed by a "unit code" (five bits ending in " 0 ") or a device command (five bits ending in " 1 "). All messages in X10 are transmitted twice to guarantee delivery as there is no acknowledgement possible from the receiver.

A similar low bit rate protocol could be used for communicating with an embedded neural receiving device. The front end of such a receiver would be a cuff electrode that would detect the CAP pulses [47]. The receiver itself could be, for example, an implantable CMOS drug delivery device, like that described by Huang et al [16]. Their drug delivery system amplifies and decodes wireless OOK signals in order to determine a specific drug reservoir location and then releases the drug by heating and melting the reservoir cap. Our CAP OOK transmission system could activate a similar embedded drug delivery unit with multiple drugs or multiple concentrations of the same drug.

\section{CONCLUSIONS}

We have described and modelled a neural serial communications channel that uses compound action potentials (CAPS) as data pulses to communicate with a receiver placed further along the nerve. The maximum achievable range between transmitter and receiver depends critically on the on the number and diameters of the activated neurons (contributing to the CAP amplitude and width) and the level of background neural noise. It would be possible to improve on the transmission range by increasing the intensity of the applied stimulus and creating a CAP of higher amplitude, assuming that not all available neurons of larger diameter had been activated. If all larger diameter neurons have been activated then any further increase in the applied stimulus intensity will trigger smaller diameter, lower velocity neurons resulting in a greater broadening of the CAP without necessarily increasing the amplitude (as shown previously in Fig 5).

We examined the two modulation methods of OOK and DPIM and concluded that OOK was the only feasible method given the timing constraints imposed by the neural channel on the DPIM inter-pulse interval. The OOK bit rate is limited by the refractory period of the neurons and results in a maximum data rate of $200 \mathrm{bits} / \mathrm{s}$. Transmission ranges of over $100 \mathrm{~mm}$ at this rate can be achieved at higher levels of SNR. Preliminary calibration of the transmission array would be necessary in order to estimate the CAP width and intensity (amplitude) at different points along the nerve before the placement of the receiving module. The calibration would provide the operating parameters needed to determine the achievable range of the maximum bit rate.

We have given the example of how an embedded drugdelivery system could be activated and programmed by CAP data pulses. There is also the possibility of sending OOK pulses along the vagus nerve, a nerve that is accessible at the neck and is one of the cranial nerves that routes directly to the brain rather than via the spinal cord. Our neural transmission system could send commands to an embedded programmable neural prosthesis in the brain to help mitigate impaired brain functions. Ideally the implanted device should have a long-life biocompatible power harvesting system that does not depend on external ultrasound or EM wireless powering. We propose the use of a glucose-based biofuel cell for powering the implant similar to that described by Rapoport et al [48]. These scenarios will be the subject of further study.

\section{REFERENCES}

[1] J. DeSantana, D. Walsh, C. Vance, B. Rakel, and K. Sluka, "Effectiveness of transcutaneous electrical nerve stimulation for treatment of hyperalgesia and pain," Current Rheumatology Reports, vol. 10(6), pp. 492-9, 2008.

[2] R. H. Howland, "Vagus nerve stimulation," Current Behavioral Neuroscience Reports, vol. 1, no. 2, Jun. 2014.

[3] F. A. Koopman, S. S. Chavan, S. Miljko, S. Grazio, S. Sokolovic, P. R. Schuurman, A. D. Mehta, Y. A. Levine, M. Faltys, R. Zitnik, K. J. Tracey, and P. P. Tak, "Vagus nerve stimulation inhibits cytokine production and attenuates disease severity in rheumatoid arthritis," Proceedings of the National Academy of Sciences, vol. 113, no. 29, pp. 8284-8289, 2016.

[4] D. Martinez-Ramirez, W. Hu, A. R. Bona, M. S. Okun, and A. W. Shukla, "Update on deep brain stimulation in parkinson's disease," Translational Neurodegeneration, vol. 4, no. 1, p. 12, Jun 2015.

[5] M. P. Natalie Hanisch, "Digital modulation and achievable information rates of thru-body haptic communications," pp. 10206 - 10206 - 13, 2017.

[6] D. Malak and O. B. Akan, "A communication theoretical analysis of synaptic multiple-access channel in hippocampal-cortical neurons," IEEE Transactions on Communications, vol. 61, no. 6, pp. 2457-2467, June 2013.

[7] E. Balevi and O. B. Akan, "A physical channel model for nanoscale neuro-spike communications," IEEE Transactions on Communications, vol. 61, no. 3, pp. 1178-1187, March 2013.

[8] H. Ramezani and O. B. Akan, "Information capacity of vesicle release in neuro-spike communication," IEEE Communications Letters, vol. 22 no. 1, pp. 41-44, Jan 2018. 
[9] M. Veletić, P. A. Floor, Y. Chahibi, and I. Balasingham, "On the upper bound of the information capacity in neuronal synapses," IEEE Transactions on Communications, vol. 64, no. 12, pp. 5025-5036, Dec 2016.

[10] A. S. Cacciapuoti, A. Piras, and M. Caleffi, "Modeling the dynamic processing of the presynaptic terminals for intrabody nanonetworks," IEEE Transactions on Communications, vol. 64, no. 4, pp. 1636-1645, April 2016.

[11] H. Ramezani, T. Khan, and O. B. Akan, "Sum rate of miso neurospike communication channel with constant spiking threshold," IEEE Transactions on NanoBioscience, vol. 17, no. 3, pp. 342-351, July 2018.

[12] A. Khodaei and M. Pierobon, "An intra-body linear channel mode based on neuronal subthreshold stimulation," in 2016 IEEE International Conference on Communications (ICC), May 2016, pp. 1-7.

[13] — "Subthreshold linear modeling of dendritic trees: A computational approach," in 2016 38th Annual International Conference of the IEEE Engineering in Medicine and Biology Society (EMBC), Aug 2016, pp. 235-238.

[14] J. Malmivuo and R. Plonsey, Bioelectromagnetism - Principles and Applications of Bioelectric and Biomagnetic Fields. Oxford University Press, 1995

[15] N. A. Abbasi, D. Lafci, and O. B. Akan, "Controlled information transfer through an in vivo nervous system," Scientific Reports, 2018.

[16] Y.-J. Huang, H.-H. Liao, P.-L. Huang, T. Wang, Y.-J. Yang, Y.-H. Wang, and S.-S. Lu, "An implantable release-on-demand cmos drug delivery soc using electrothermal activation technique," J. Emerg. Technol. Comput. Syst., vol. 8, no. 2, pp. 12:1-12:22, Jun. 2012.

[17] M. Donohoe, S. Balasubramaniam, B. Jennings, and J. M. Jornet, "Powering in-body nanosensors with ultrasounds," IEEE Transactions on Nanotechnology, vol. 15, no. 2, pp. 151-154, March 2016.

[18] M. Donohoe, B. Jennings, J. M. Jornet, and S. Balasubramaniam, "Nanodevice arrays for peripheral nerve fascicle activation using ultrasound energy-harvesting," IEEE Transactions on Nanotechnology, vol. 16 , no. 6, pp. 919-930, Nov 2017

[19] Y. Qin, M. M. Howlader, M. J. Deen, Y. M. Haddara, and P. R Selvaganapathy, "Polymer integration for packaging of implantable sensors," Sensors and Actuators B: Chemical, vol. 202, pp. $758-778$, 2014.

[20] D. T. Brocker and W. M. Grill, "Chapter 1 - principles of electrical stimulation of neural tissue," in Brain Stimulation, ser. Handbook of Clinical Neurology, A. M. Lozano and M. Hallett, Eds. Elsevier, 2013 , vol. 116 , pp. $3-18$

[21] M. D. Mann, The Nervous System in Action. http://michaeldmann.net/The Nervous System In Action.html, 2018.

[22] S. Nandedkar and E. Stalberg, "Simulation of single muscle fibre action potentials," Medical and Biological Engineering and Computing, vol. 21 pp. 158-165, 1983.

[23] P. Rosenfalck, "Intra and extracellular fields of active nerve and muscle fibers. a physico-mathematical analysis of different models." Acta Physiologica Scandinavica, 1969.

[24] S. Rutkove, "Introduction to volume conduction," in The Clinical Neurophysiology Primer. Humana Press, 2007.

[25] R. Plonsey, "The active fiber in a volume conductor," IEEE Transactions on Biomedical Engineering, vol. BME-21, no. 5, pp. 371-381, Sept 1974.

[26] N. A Dimitrova, A. G. Dimitrov, and G. V. Dimitrov, "Calculation of extracellular potentials produced by an inclined muscle fibre at a rectangular plate electrode," Medical Engineering \& Physics, vol. 21, no. 8, pp. 583 - 588, 1999.

[27] J. R. Falces, A. M. Trigueros, L. G. Useros, I. R. Carreno, and J. N. Irujo, "A mathematical analysis of sfap convolutional models," IEEE Transactions on Biomedical Engineering, vol. 52, no. 5, pp. 769-783, May 2005

[28] J. Struijk, "The extracellular potential of a myelinated nerve fiber in an unbounded medium and in nerve cuff models," Biophysical Journal, vol. 72 , no. 6 , pp. $2457-2469,1997$.

[29] S. Joucla and B. Yvert, "Modeling extracellular electrical neural stimulation: From basic understanding to mea-based applications," Journal of Physiology-Paris, vol. 106, pp. 146 - 158, 2012, neuronal Ensemble Recordings in Integrative Neuroscience.

[30] R. S. Wijesinghe, F. L. H. Gielen, and J. P. Wikswo, "A model for compound action potentials and currents in a nerve bundle i: The forward calculation," Annals of Biomedical Engineering, vol. 19, no. 1, pp. 43$72,1991$.

[31] R. Schoonhoven, D. F. Stegeman, and J. P. C. D. Weerd, "The forward problem in electroneurography $\mathrm{i}$ : A generalized volume conductor model," IEEE Transactions on Biomedical Engineering, vol. BME-33, no. 3, pp. 327-334, March 1986.

[32] L. J. Snyder A.W., "Pulse spreading," in Optical Waveguide Theory. Springer, Boston, MA, 1983.

[33] D. F. Stegeman, R. Schoonhoven, G. F. M. Dautzenberg, and J. Moleman, "The inverse problem in electroneurography. ii. computational aspects and evaluation using simulated data," IEEE Transactions on Biomedical Engineering, vol. 35, no. 10, pp. 778-788, Oct 1988.

[34] K. Guillory and R. Normann, "A 100-channel system for real time detection and storage of extracellular spike waveforms," Journal of Neuroscience Methods, vol. 91, no. 1, pp. 21 - 29, 1999.

[35] R. R. Harrison, "A low-power integrated circuit for adaptive detection of action potentials in noisy signals," in Proceedings of the 25th Annual International Conference of the IEEE Engineering in Medicine and Biology Society (IEEE Cat. No.03CH37439).

[36] M. Lewicki, "Bayesian modeling and classification of neural signals," Neural Computation, 1994

[37] A. Diedrich, W. Charoensuk, R. J. Brychta, A. C. Ertl, and R. Shiavi, "Analysis of raw microneurographic recordings based on wavelet denoising technique and classification algorithm: wavelet analysis in microneurography," IEEE Transactions on Biomedical Engineering, vol. 50, no. 1, pp. 41-50, Jan 2003.

[38] J. Martinez, C. Pedreira, M. J. Ison, and R. Q. Quiroga, "Realistic simulation of extracellular recordings," Journal of Neuroscience Methods, vol. 184 , no. 2, pp. $285-293$, 2009

[39] C. E. Shannon, "Communication in the presence of noise," Proceedings of the IRE, vol. 37, no. 1, pp. 10-21, Jan 1949.

[40] R. L. Freeman, "Bits, symbols, bauds, and bandwidth," IEEE Communications Magazine, vol. 36, no. 4, pp. 96-99, Apr 1998

[41] Z. Ghassemlooy, A. R. Hayes, N. L. Seed, and E. D. Kaluarachchi, "Digital pulse interval modulation for optical communications," IEEE Communications Magazine, vol. 36, no. 12, pp. 95-99, Dec 1998.

[42] B. Krishnaswamy, C. M. Austin, J. P. Bardill, D. Russakow, G. L Holst, B. K. Hammer, C. R. Forest, and R. Sivakumar, "Time-elapse communication: Bacterial communication on a microfluidic chip," IEEE Transactions on Communications, vol. 61, no. 12, pp. 5139-5151, December 2013

[43] M. T. Barros, S. Balasubramaniam, B. Jennings, and Y. Koucheryavy, "Transmission protocols for calcium-signaling-based molecular communications in deformable cellular tissue," IEEE Transactions on Nanotechnology, vol. 13, no. 4, pp. 779-788, July 2014

[44] D. M. MacKay and W. S. McCulloch, "The limiting information capacity of a neuronal link," The bulletin of mathematical biophysics, vol. 14 no. 2 , pp. $127-135,1952$.

[45] L. J. Ippolito, Appendix B: Error Functions and Bit Error Rate. John Wiley \& Sons, Ltd, 2008, pp. 363-366.

[46] M. D. Cruzl, J. A. Ortegal, Á. Barriga, and A. Fernández-Montes, "Development environment using fpga for domotics applications based on x10 technology," in Novel Algorithms and Techniques In Telecommunications, Automation and Industrial Electronics, T. Sobh, K. Elleithy, A. Mahmood, and M. A. Karim, Eds. Dordrecht: Springer Netherlands, 2008, pp. 150-153.

[47] N. S. Korivi and P. K. Ajmera, "Clip-on micro-cuff electrode for neural stimulation and recording," Sensors and Actuators B: Chemical, vol. 160, no. 1, pp. 1514 - 1519, 2011.

[48] B. I. Rapoport, J. T. Kedzierski, and R. Sarpeshkar, "A glucose fuel cell for implantable brainmachine interfaces," PLOS ONE, vol. 7, no. 6, pp. $1-14,062012$ 


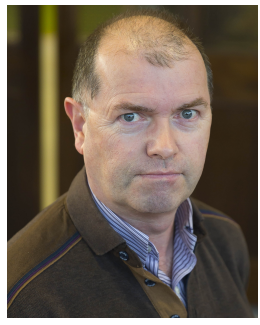

Michael Donohoe (M16) received a BSc in Physics from University College Galway, Ireland, in 1980. $\mathrm{He}$ is at present a $\mathrm{PhD}$ student in the Telecommunications Software and Systems Group (TSSG) of the Waterford Institute of Technology, Ireland. Prior to this he worked in the telecommunications industry especially in the areas of network planning, project implementation, R\&D and product management. His research interests include embedded nanodevices, energy harvesting and in-body communications networks.

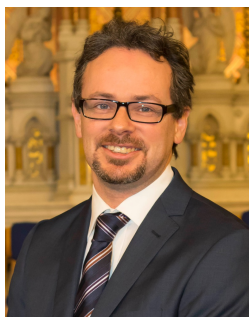

Brendan Jennings (M05) received the BEng and $\mathrm{PhD}$ degrees from Dublin City University, Dublin, Ireland, in 1993 and 2001, respectively. He is the Head of Graduate Studies for the Waterford Institute of Technology, Ireland and a Principal Investigator with CONNECT, Irelands national centre for communications networking research. He has spent periods as a Visiting Researcher with the KTH Royal Institute of Technology, Sweden, and in EMC Research Europe, Ireland. He regularly serves on the organization and technical program committees of a number of network and service management related conferences. He will serve as Executive Chair for the IEEE ICC 2020 conference, to be held in Dublin, Ireland in June 2020. His research interests include network management, cloud computing, and nanoscale communications.

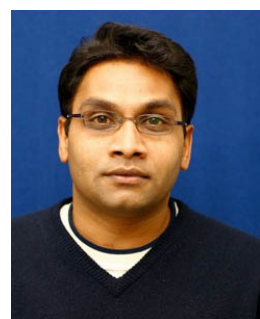

Sasitharan Balasubramaniam (SM14) received his Bachelor (Electrical and Electronic Engineering) and Ph.D. degrees from the University of Queensland in 1998 and 2005, respectively, and Masters (Computer and Communication Engineering) degree in 1999 from the Queensland University of Technology. He is currently an Academy of Finland Research Fellow at the Department of Electronic and Communication Engineering, Tampere University of Technology, Finland, and Acting Director of Research at the Telecommunication Software and Systems Group, Waterford Institute of Technology, Ireland, where he worked on a number of Science Foundation Ireland projects. Sasitharan is in the Steering Committee of the ACM NanoCom conference which he co-founded. In 2018 he received the ACM/IEEE NanoCom Outstanding Milestone award, and he is also the IEEE Nanotechnology Council Distinguished Lecturer. He is currently an editor for the IEEE Internet of Things journal, Elsevier Nano Communication Networks, and Elsevier Digital Communication Networks. His current research interests includes molecular and nano communications, and Internet of (Bio-Nano) Things. 